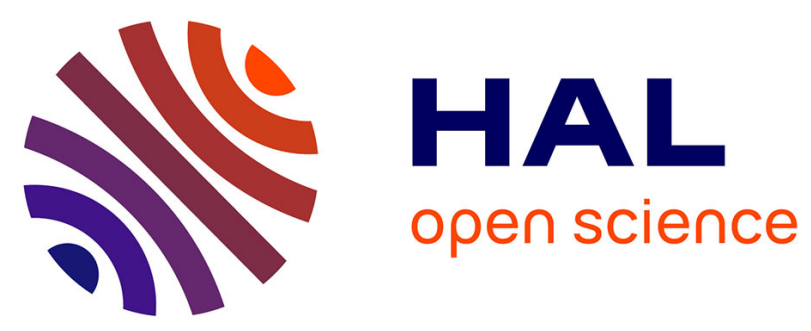

\title{
A sunflower BAC library suitable for PCR screening and physical mapping of targeted genomic regions
}

Mohamed Fouad Bouzidi, Jérôme Franchel, Quanzhou Tao, Keith Stormo, A. Mraz, Paul Nicolas, Saïd S. Mouzeyar

\section{- To cite this version:}

Mohamed Fouad Bouzidi, Jérôme Franchel, Quanzhou Tao, Keith Stormo, A. Mraz, et al.. A sunflower BAC library suitable for PCR screening and physical mapping of targeted genomic regions. TAG Theoretical and Applied Genetics, 2006, 113 (1), pp.81-89. 10.1007/s00122-006-0274-6 . hal02147849

\section{HAL Id: hal-02147849 \\ https://hal.science/hal-02147849}

Submitted on 5 Jun 2019

HAL is a multi-disciplinary open access archive for the deposit and dissemination of scientific research documents, whether they are published or not. The documents may come from teaching and research institutions in France or abroad, or from public or private research centers.
L'archive ouverte pluridisciplinaire HAL, est destinée au dépôt et à la diffusion de documents scientifiques de niveau recherche, publiés ou non, émanant des établissements d'enseignement et de recherche français ou étrangers, des laboratoires publics ou privés. 


\section{A sunflower BAC library suitable for PCR screening and physical mapping of targeted genomic regions}

\begin{abstract}
A sunflower BAC library consisting of 147,456 clones with an average size of $118 \mathrm{~kb}$ has been constructed and characterized. It represents approximately $5 \times$ sunflower haploid genome equivalents. The BAC library has been arranged in pools and superpools of DNA allowing screening with various PCR-based markers. Each of the 32 superpools contains 4,608 clones and corresponds to a 36 matrix pools. Thus, the screening of the entire library could be accomplished in less than 80 PCR reactions including positive and negative controls. As a demonstration of the feasibility of the concept, a set of 24 SSR markers covering about $36 \mathrm{cM}$ in the sunflower SSR map (Tang et al. in Theor Appl Genet 105:1124-1136, 2002) have been used to screen the BAC library. About $125 \mathrm{BAC}$ clones have been identified and then organized in 23 contigs by HindIII digestion. The contigs are anchored on the SSR map and thus constitutes a first-generation physical map of this region. The utility of this BAC library as a genomic resource for physical mapping and map-based cloning in sunflower is discussed.
\end{abstract}

M. F. Bouzidi · J. Franchel · P. Nicolas · S. Mouzeyar $(\bowtie)$

UMR 1095 INRA-UBP “Amélioration et Santé des Plantes", Université Blaise Pascal, 24 avenue des Landais, 63177 Aubière Cedex, France

E-mail: Said.MOUZEYAR@univ-bpclermont.fr Tel.: +33-4-73407911

Fax: +33-4-73407914

Q. Tao $\cdot$ K. Stormo $\cdot$ A. Mraz

Amplicon Express, 2345 NE Hopkins Court, Pullman, WA 99163, USA

\section{Introduction}

The sunflower, Helianthus annuus L. is one of the major crops for oil source and is widely cultivated throughout the globe. It is a diploid plant with an estimated haploid genome size of about $3,000 \mathrm{Mb}$ and $2 n=2 x=34$ chromosomes (Arumuganathan and Earle 1991). Many genomic resources have been developed for this plant including genetic maps based either on anonymous RFLP markers (Berry et al. 1995; Gentzbittel et al. 1995, 1999) or more recently on microsatellite markers (Tang et al. 2002). These genetic maps are an invaluable resource that can be used not only to map even complex traits such as QTL but also to anchor physical maps (Yim et al. 2002). In addition, a massive effort is currently made for the release of a vast collection of sunflower ESTs and currently more than 66,000 sunflower ESTs are available in the public databases. These sequences constitute a valuable source for the direct access to some genes of interest or for the development of molecular markers for map-based cloning purposes or marker-assisted selection programs. However, the link between these markers and their physical location on the sunflower genome needs to be developed. With this respect, two sunflower BAC libraries have been described recently. A BAC library made up of 158,208 clones and arrayed on nine nylon filters has been described by Gentzbittel et al. (2002) and a second BAC library containing about 100,000 clones and covering $1.9 \times$ haploid sunflower genome has been described by Ozdemir et al. (2004). However, both libraries were not designed for systematic and high throughput anchoring of PCR markers on BAC clones. In this paper we describe the design and the construction of a sunflower BAC library suitable not only for southern hybridization but also for PCR screening. Less than 80 PCR reactions are needed to detect one positive $\mathrm{BAC}$ clone containing a given PCR-based marker. Furthermore, the pooling and superpooling methods described in this paper could be used for the construction of BAC libraries even from large genomes. We have used this concept to screen the BAC library, with a set of 25 SSR markers covering about $36 \mathrm{cM}$ in the sunflower SSR map (Tang et al. 2002). About 112 BAC clones have been identified and then organized in 23 contigs by HindIII digestion. The contigs which correspond roughly to $3.1 \mathrm{Mb}$ are 
anchored on the SSR map and thus constitutes a firstgeneration physical map of this region.

\section{Materials and methods}

\section{Sunflower genotype}

The sunflower line YDQ was used for the construction of the BAC library. This line was bred by INRA (Clermont-Ferrand, France) and is resistant to several races of the biotrophic parasite Plasmopara halstedii (Bouzidi et al. 2002).

\section{BAC library construction}

This sunflower BAC library was constructed from high molecular weight (HMW) genomic DNA processed at Amplicon Express, Pullman, Washington using the method of Tao et al. (2002). DNA digestion was done with varying amounts of HindIII to identify an appropriate partial digestion condition. The vector used was derived from the pBeloBAC11 vector and contains two $F R T$ and one oriV elements, thus resulting in the pBAC $(F R T$-oriV $)$ vector. Ligations were transformed into DH10B Escherichia coli cells (Invitrogen) and plated on LB agar with appropriate chloramphenicol, X-gal and IPTG concentrations. Clones were robotically picked with a Genomic Solution G3 into 384 well plates containing LB freezing media. Plates were incubated for $18 \mathrm{~h}$, replicated and then frozen at $-80^{\circ} \mathrm{C}$. The replicated copy was used as a source plate for printing nylon filters and for inoculating plates for pooling.

\section{Insert size estimation}

To estimate insert sizes, $10 \mu \mathrm{l}$ aliqots of BAC miniprep DNA were digested with $5 \mathrm{U}$ of Not I enzyme during $3 \mathrm{~h}$ at $37^{\circ} \mathrm{C}$. The digestion products were separated by pulsed field gel electrophoresis (CHEF-DRIII system, Bio-rad) in a $1 \%$ agarose gel in TBE buffer. Insert sizes were compared to those of the Lambda Ladder PFG Marker (New England Biolabs). Electrophoresis was carried out for $18 \mathrm{~h}$ at $14^{\circ} \mathrm{C}$ with an initial switch time of $5 \mathrm{~s}$, a final switch time of $15 \mathrm{~s}$, in a voltage gradient of $6 \mathrm{~V} / \mathrm{cm}$.

Filter design and construction

Bioassay dishes containing LB agar plate media and $12.5 \mu \mathrm{g} / \mathrm{ml}$ chloramphenicol were prepared. Hybond $\mathrm{N}^{+}$ (Amersham, Biosciences) nitrocellulose membrane was applied to the media surface. The G3 robot was used to grid 18,432 clones in duplicate on each filter following a $4 \times 4$ pattern that allows the well position and plate number of each positive clone to be identified. The filters were incubated at $37^{\circ} \mathrm{C}$ for $12-14 \mathrm{~h}$ and processed by a slightly modified nylon filter lysis method. The filters were fixed according to the Hybond $\mathrm{N}^{+}$manual by baking at $80^{\circ} \mathrm{C}$ for $2 \mathrm{~h}$.
Sunflower pooling and superpooling method

The pooling strategy chosen (Fig. 1) was the standard Matrix Pool and Superpool Strategy by Amplicon Express. The strategy consists of a two-step approach in which the entire BAC library of 147,456 clones on 384 microtiter plates is arranged into 32 superpools, each composed of 12 sequential 384 well plates. The clones in these 12 plates are systematically combined into plate, row, column and diagonal (PRCD) pools. These PRCD pools are further combined following the Matrix Technology Strategy, which reduces the number of pools to be screened in the second step from 76 (PRCD) to 36 (Matrix) for each superpool. Each superpool contains 4,608 unique BAC clones with an average insert size of $118 \mathrm{~kb},(\sim 0.18 \times$ coverage of the $3,000 \mathrm{Mb}$ haploid sunflower genome). Therefore, it is expected that zero or one template copy of any single copy sequence will be found in a specific Superpool.

Screening the pools and superpools involves two steps: step one is to identify which of the 32 superpools contain a signal for the sequence of interest. Step two is to screen the 36 matrix pools for as many superpools identified in step one as the researcher wants to pursue. For quality control purposes, an internal standard plasmid or total genomic DNA is placed in one of the empty wells of the BAC library. The plate pool that contains this positive control clone is also placed in one well of each PRCD plate to facilitate quality control verification and PCR positive control for each superpool.

Each BAC clone was grown independently to prevent growth competition between clones in microtiter plates containing $2 \mathrm{xYT}$ broth with $12.5 \mu \mathrm{g} / \mathrm{ml}$ chlorampheni$\mathrm{col}$. The plates were incubated for $16 \mathrm{~h}$ at $37^{\circ} \mathrm{C}$. Following growth, the culture for each set of 12 plates was pooled using the method described. Following pooling, sequencing grade BAC DNA preparation was performed on all pooled clones in 96 well format deep blocks using an optimized alkaline lysis method. BAC plasmid DNA was resuspended in $200 \mu \mathrm{l} \mathrm{TE}(\sim 20 \mathrm{ng} / \mu \mathrm{l})$, then pooled to create the Matrix Master pools.

Pooling of BAC clone cultures was performed on a Biomek 2000 liquid handling robot with customized software and hardware. The growth cultures were combined into 12 plate pools, 16 row pools, 24 column pools and 24 diagonal pools for each superpool. The coordinates of any positive BAC clone can be identified by interpreting the resulting PCR data from experiments using superpool, plate pool, row pool, column pool and diagonal pool DNA as template. The diagonal pools help reduce the number of potential false positive clones when multiple positive clones are contained in a single superpool.

Extraction of BAC DNA occurs on the intermediate stage of the PRCD pools. The BAC DNA from these PRCD pools is further pooled so that each PRCD pool is contained in two matrix pools. Each individual clone has eight occurrences in the matrix pool set (for each superpool), allowing a smaller number of PCR experiments 
to correctly identify the unique well position in each superpool, and providing redundant results to minimize the impact of false negative and false positive PCR results. See Fig. 1 for the composition of the 36 matrix pool.

Superpooled and matrix pooled BAC DNA is prepared as a high concentration master stock and as a diluted PCR working stock, allowing a backup master stock to be kept in case of accidental contamination of the working stock. The highly concentrated resource can also be used in a variety of analytical techniques. All 32 superpools have an individual matrix pool plate. The superpool collection plate contains DNA from the entire library with 4,608 clones in each superpool well. The matrix pools and superpools are contained in $1 \mathrm{ml}$ half height plates sealed with a silicone mat.

\section{Screening of the BAC library}

Filter hybridization: filters were hybridized using standard protocols. The probes were labeled with $\left[{ }^{32} \mathrm{P}\right]$-dCTP by random priming according to the manufacturer's instructions (Amersham-Pharmacia Biotech). Filters were prehybridized for $4 \mathrm{~h}$ at $65^{\circ} \mathrm{C}$ in a solution containing $1 \times$ HSB, $5 \times$ Denhardt's buffer and salmon sperm DNA. They were then hybridized overnight in the same solution at $65^{\circ} \mathrm{C}$ but containing the labeled probes. Filters were then washed at $65^{\circ} \mathrm{C}$ in a $2 \times$ SSC and $0.1 \%$ SDS solution for $15 \mathrm{~min}$, then in a $1 \times \mathrm{SSC}$ and $0.1 \%$ SDS solution for $15 \mathrm{~min}$, and finally in a $0.1 \times \mathrm{SSC}$ and $0.1 \%$ SDS solution for $15 \mathrm{~min}$. The filters were then exposed to X-ray films with two intensifying screens.

\section{PCR screening}

The PCR amplifications were carried out with $2 \mu 1$ of each template in the presence of $0.2 \mathrm{mM}$ of each dNTP, $0.2 \mu$ l of Taq DNA polymerase (Advantage 2, Clontech), $1 \times$ Taq polymerase buffer and $1 \mu \mathrm{M}$ of each primer, in a final volume of $20 \mu \mathrm{l}$. Following initial denaturation at $95^{\circ} \mathrm{C}$ for $5 \mathrm{~min}, 35 \mathrm{PCR}$ cycles of $95^{\circ} \mathrm{C}$ for $30 \mathrm{~s}, 60^{\circ} \mathrm{C}$ for



Fig. 1 Schematic representation of the pooling strategy. Twelve 384-well plates are pooled to form a superpool $(S P)$. The entire library consists of 32 superpools. Each of the 32 superpools corresponds to a matrix pool containing pools of Plates, Rows, Columns and Diagonals as depicted in the figure. Examples are given (in grey) for the Plate pool "P1:1,2,3", the Row pool "R8:D,H,L,P", the Column pool "C8:4,10,16,22" and the Diagonal pool "D5: 1,7,13,19". The black circles indicate the clones that have been mixed to make up each pool. Positive control contains DNA from the sunflower line YDQ. Negative control contains no DNA 
$30 \mathrm{~s}$ and $72^{\circ} \mathrm{C}$ for $1 \mathrm{~min}$ were performed. PCR products were separated using $2 \%$ standard TAE agarose gel electrophoresis.

\section{BAC fingerprinting and contig assembly}

BAC DNA were isolated and digested with HindIII, endlabeled with $\left[{ }^{32} \mathrm{P}\right] \mathrm{dATP}$ and then subjected to $3.5 \%$ polyacrylamide DNA sequencing gel electrophoresis. The gels were dried and autoradiographed. The fingerprints on the autoradiographs were scanned into images and edited using the Image 4.0 software (Soderlund et al. 1997). The BAC contigs were assembled using the version 4.7 of the FPC program (Soderlund et al. 2000).

\section{Results}

\section{BAC library construction and characterization}

The present library consists of 147,456 BAC clones and is represented by a total of 384 384-well microtiter plates. In order to evaluate the average $\mathrm{BAC}$ insert sizes, 297 clones were randomly chosen and the corresponding DNA was extracted, digested with the rarecutter NotI enzyme and analyzed by PFGE (Fig. 2a). All fragments generated by NotI digestion contained $8.7 \mathrm{~kb}$ vector band and various insert fragments, except six presumed to be non-insert clones. The estimated insert sizes ranged from 40 to $210 \mathrm{~kb}$ with an average of $118 \mathrm{~kb}$ (Fig. 2b). The haploid genome size of the cultivated sunflower $H$. annuus $\mathrm{L}$. is estimated to be 2,800 $3,400 \mathrm{Mb}$ (Arumuganathan and Earle 1991), hence the coverage of this library is predicted to be 5-6 haploid genome equivalents, and thereby the probability of finding any specific sequence in this library is estimated to be greater than $99 \%$.

\section{PCR screening of the library}

To test the feasibility of the pooling scheme used for this library, we used one primer pair for PCR screening. The primers amplify a partial fragment of about $150 \mathrm{bp}$ of Ha-PR 5 gene in sunflower (Radwan et al. 2005). The first PCR round detected two superpools (16 and 28) with positive amplification compared to the signal detected using as template the DNA from the parent from which the library was constructed (Fig. 3a). The corresponding PRCD pools were subsequently used in a second PCR round (Fig. 3b). The example presented corresponds to the matrix pools of the superpool number 28 . Thus, the PRCD coordinates of this particular clone are respectively: plate number 336 in the library, Row: N, Column 16 and Diagonal 5 which contains the 16th column of the last plate in the superpool (see Fig. 1, Materials and methods section). To ensure that the amplified fragments correspond to the initial target, these fragments were
Fig. 2 Characterization of the sunflower BAC library. a Pulsed field gel electrophoresis of randomly selected BAC clones. DNA samples from 24 BAC clones were digested with NotI, and the average insert size and rate of non-recombination were determined. b Insert size distribution in the library. Insert sizes were determined on 297 randomly selected BAC clones. Six clones are non-recombinant. The horizontal axis refers to the size in $\mathrm{kb}$ and vertical axis indicates the number of clones in each size range. $M$ refers to the high range PFG markers (New England Biolabs). The $8.7 \mathrm{~kb}$ band corresponds to the plasmid vector
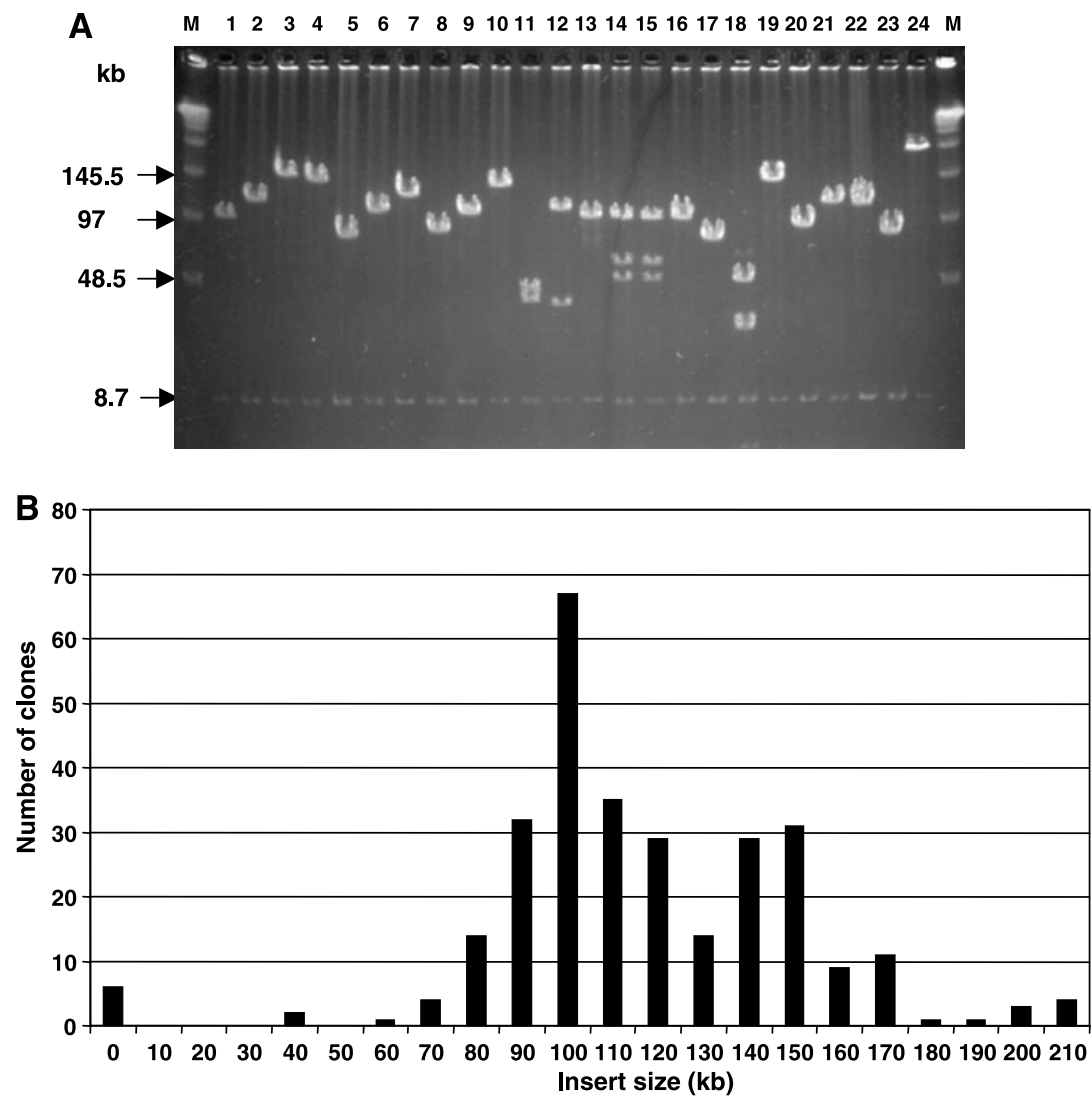
Fig. 3 PCR screening of the pools and superpools of the sunflower library. a First round PCR using the 32 superpools which represents the entire library or 384 384-well microtiter plates. Each superpool contains DNA of 12 plates or 4,608 individual BAC DNAs. Two superpools (16 and 28) contain a signal for Ha-PR 5 gene coding for the pathogenesis-related protein 5. b Second round PCR using the 36 matrix pools to screen the superpool number 28 identified in step one. The clones in the 36 matrix pools are systematically combined into Plate (X 8), Row (X 8), Column (X 10) and Diagonal (X 10) (PRCD) pools. Sunflower genomic DNA from the line YDQ represents the positive control $(Y D Q) . W$ indicates the negative controls (water replaced BAC DNA)
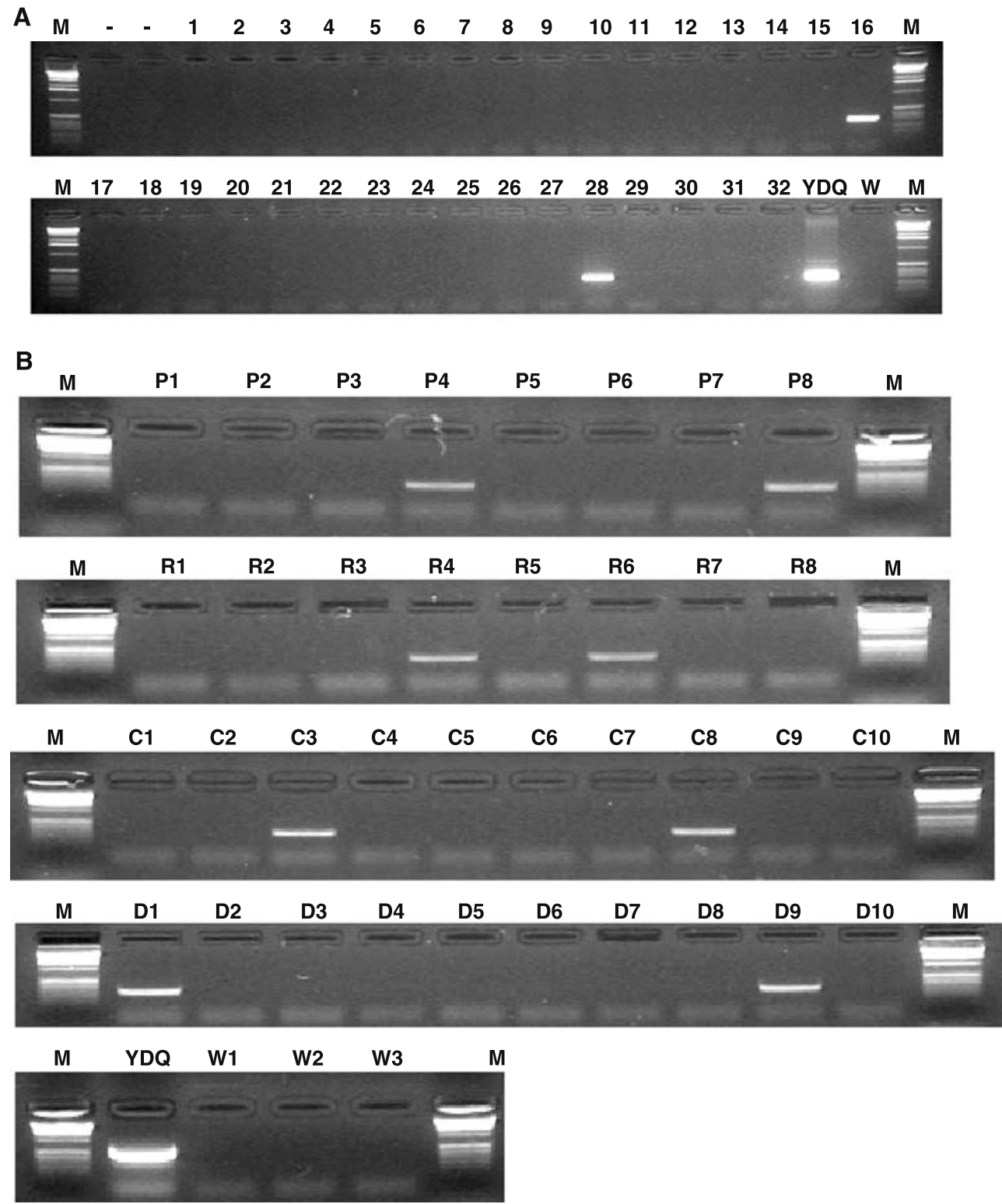

cloned and sequenced. The sequences obtained correspond exactly to the one from which the primers were derived, indicating that the different clones are containing the same target sequence. Subsequent fingerprinting of the two BAC clones by HindIII digestion proved that they are overlapping.

Anchoring the BAC clones on the SSR map of sunflower

To test whether this strategy could be widely used for different PCR markers, we used a set of SSR markers to screen the library. One region highly covered by SSR markers was selected from the sunflower SSR map described by Tang et al. (2002). Twenty-five SSR markers detecting unique loci within the linkage group 10 of this map were used to screen the BAC library. All the SSR markers detected at least one positive BAC clone in the library. A total of 113 BAC clones were identified and the number of BAC clones detected by each SSR marker ranged from 1 to 8 giving on average $4.8 \mathrm{BAC}$ clones per SSR marker. This is in the range of the estimated 5-6 redundancy. Conversely, no BAC clone was found to harbor two different SSR markers. These results are summarized in Fig. 4.

Fingerprinting of the positive $\mathrm{BAC}$ clones

Since several BAC clones were detected by the same SSR marker, it was questionable whether these BAC clones are identical or overlapping. All the BAC clones (i.e., 112 BAC clones) were thus HindIII digested and fingerprinted using various FPC iterations and parameters (Soderlund et al. 2000). The results are given in Fig. 4. As it was expected, the majorities of the BAC clones that share the same SSR marker are overlapping and thus belong to the same BAC contig. This is consistent with 

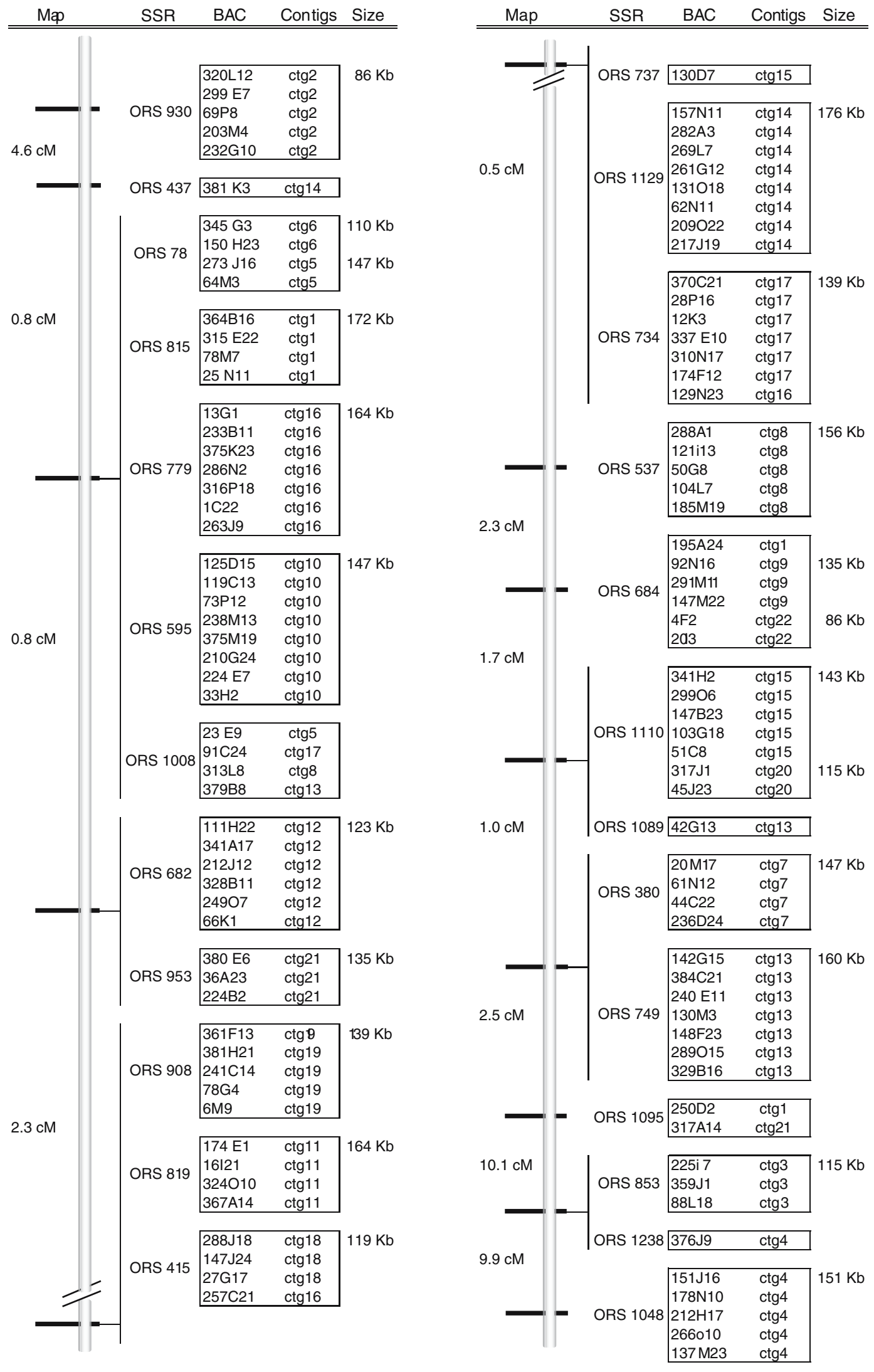
Fig. 4 First-generation physical map of a $36 \mathrm{cM}$ region in sunflower genome. Twenty-five SSR markers detecting unique loci within the linkage group 10 from the sunflower SSR map described by Tang et al. (2002) were used to screen the BAC library. All the SSR markers detected at least one positive BAC clone in the library. A total of $113 \mathrm{BAC}$ clones were identified and arranged in 23 contigs. The number of BAC clones detected by each SSR marker ranged from 1

the fact that the SSR markers used in this study correspond to single mapped loci (Tang et al. 2002). Seven SSR markers detected three or more BAC clones with predicted overlaps that have been confirmed by fingerprinting analysis. For example, the SSR marker ORS 595 identified eight $\mathrm{BAC}$ clones that formed the unique contig 10. In contrast, some of the BAC clones that share the same SSR marker were found within different contigs even when the cutoff value of 10 was used (low fingerprinting stringency, Soderlund et al. 2000). For example, the ORS 78 SSR marker detected four positive BAC clones which were placed by fingerprinting in two distinct contigs.

\section{Discussion}

\section{BAC library construction and characterization}

We have constructed a sunflower BAC library consisting of 147,456 clones with an average insert size of $118 \mathrm{~kb}$, thus representing approximately 5-6 sunflower haploid genome equivalents. The insert size of around $100-200 \mathrm{~kb}$ makes it an ideal source for physical mapping, FISH analysis to localize the position on chromosomes, isolation of closely linked polymorphic markers, exon trapping, and identification of regulatory elements in gene expression studies (Buckler et al. 1999; Ota and Amemiya 1996).

The clones were produced using HindIII partially digested DNA from nuclei of the sunflower line YDQ which is resistant to several races of the sunflower downy mildew pathogen P. halstedii (Bouzidi et al. 2002). The BAC clones were arrayed on high-density filters for hybridization with radiolabeled probes and several screening by different probes have been successfully carried out (data not shown). A sunflower BAC library with comparatively similar characteristics (i.e., 5- to 6-fold and contains about 150,000 BAC clones) has been previously described by Gentzbittel et al. (2002). A second BAC library with $60 \mathrm{~kb}$ as average insert length and covering about $1.9 \times$ sunflower haploid genome has been reported by Ozdemir et al. (2004). However, these libraries are made up from different sunflower lines with different agronomic traits which render them complementary genomic resources for the scientific community working on this crop. Along with the production of high-density filters for colony hybridization with radioactively labeled probes, we designed a pooling and superpooling strategy to 8 giving on average 4.8 BAC clones per SSR marker. Map stands for the map position of the SSR markers as described by Tang et al. (2002). SSR stands for the SSR markers. BAC stands for the name of the BAC clone in the library. Contigs indicates the contigs generated by the FPC program after HindIII digestions. Size indicates the estimated size of the contig

suitable for large and systematic screening of this library using a wide range of PCR-based markers.

\section{Pooling and superpooling strategy}

This pooling design was based on a BAC library with 147,456 clones (average insert $118 \mathrm{~kb}$ ) in 384 384-well plates with approximately $5.8 \times$ haploid genome coverage. The Matrix pooling strategy was developed to address the following important factors. First was to minimize the number of PCR experiments required to screen the library. In this strategy, only 72 PCR reactions (32 superpools +36 matrix pools +4 positive and negative controls, see Fig. 3) are needed to detect a positive BAC clone. The second consideration in the Matrix pooling design was oversampling the pooled resource to minimize the impact of false positive or false negative PCR results. The final important design factor was to allow the researcher to perform large numbers of PCR experiments on the Pool and Superpool resource $(+20,000$ independent screenings).

Ozdemir et al. (2004) described a pooling strategy consisting of 273 plate pools, 144 column pools, and 96 row pools. Thus, 513 PCR reactions are needed to identify a particular BAC clone which is significantly higher than in the strategy proposed in this study (72 PCR reactions).

The Asakawa et al. (1997) pooling strategy used to study the human genome that consisted of 96,000 clones ( $\sim 3 \times$ haploid coverage) arranged for screening as 10 superpools with 9,600 clones in each superpool. Their pooling strategy was a two-step method where the superpool was first identified. Then the 40 pools (from fourdimensionally assigned pools of the specific superpool) were screened to identify the specific clone in the second step. Their pooling method did not include any oversampling which will always lead to significant difficulties if multiple clones, false negative or false positives occur during PCR screening.

Klein et al. (2000) used a six dimensional pooling strategy to screen 24,576 sorghum BAC clones that were about $4 \times$ haploid genome coverage. Their strategy allowed a single screen of 184 pools that would identify between two and six positive clones, $72 \%$ of the time and $90 \%$ of the time being able to reliably assign the coordinates. Their oversampling insures good identification of up to six clones and allowed screening of the complete library in one step. However, this strategy appears to have difficulty in reliably assigning coordinates if more than six positive clones are found in the library. For a large library with 147,456 clones, this strategy would 
have to be repeated at least once or performed on a large block design. For example, using a large block size of $64 \times 48 \times 48$ would have 3,072 in five of the six dimensions and 2,304 in the sixth dimension pool. This $64 \times 48 \times 48$ design would require a total of 336 pools to maintain the six dimension design. The design would have a decreased ability to identify correct clones when compared to a small block design, especially with a $5.8 \times$ coverage BAC library. With the Matrix pooling design, the same 336 PCR reactions could follow eight superpool hits in eight matrix plates to find eight positive clones with very high reliability. For example, if there were six hits in six superpools, following them all would only take a total of 248 PCRs.

\section{Screening the library by PCR-based markers}

Several different PCR markers have been successfully used to screen this BAC library (data not shown). These markers were derived either from sunflower EST representing unique genes and small multigene family, or from SSR markers which correspond to repetitive sequences within the sunflower genome (Tang et al. 2002). Nevertheless, we were able to detect specific BAC clones harboring these repetitive sequences and corresponding to unique loci in the sunflower map. The repetitive nature of these markers almost precludes their use as radiolabeled probes since they will detect several hundreds of BAC clones. For example, in a survey of the distribution of LINEs and Gypsy-like retrotranspons elements in Hordeum species, Vershinin et al. (2002) showed that in hybridization conditions where sequences with $70-75 \%$ identity will cross-hybridize, the Gypsy-like probe HvGyp13 detected up to 4,792 BAC clones. Thus, we expect that any PCR marker can be used to identify the corresponding BAC clone with an accuracy that is limited only by the specificity of the primers used. Similarly, we prepared DNA from the 32 superpools enough to achieve up to 20,000 first-round PCR screenings. Considering that the sunflower genome size is about $3,000 \mathrm{Mb}$ and sufficient PCR-based marker evenly distributed throughout the genome are provided, this amount of DNA is roughly sufficient to anchor up to one PCR-based marker every $150 \mathrm{~kb}$. This is beyond the average size of the BAC clone inserts of this library. Alternatively, PCR reactions can be multiplexed which should significantly increase the number of total possible PCR reactions. Therefore, by multiplexing three PCR reactions, it is possible to anchor up to one marker per $50 \mathrm{~kb}$ on average. However, these theoretical expectations may be out ruled by practical difficulties such as the development of such a huge number of PCR markers (Klein et al. 2000).

Rapid identification of BAC contigs within a specific region in the sunflower genome

In the present work, we presented the rapid anchoring of a set of 25 SSR markers that were previously mapped to a unique region in the sunflower genome (Tang et al.
2002). Several SSR markers were found tightly linked (i.e., $0 \mathrm{cM}$ ) during the map construction; however, none of these SSR detected the same BAC clone in our screening which indicates that at least in this region, the SSR markers were separated by more than $100 \mathrm{~kb}$ which is the average size of the BAC inserts. In addition, this distance is probably underestimated since the map was generated using a set of 94 F7 recombinant inbred lines (Tang et al. 2002) which is too small to detect all the cross-over that could happen between SSRs. Alternatively, the selected markers are likely within the centromeric region where the recombination may be severely inhibited.

The BAC clones were fingerprinted and placed in 23 contigs. The fingerprinting results were predictably consistent with the mapping data in that the majority of the BAC clones sharing the same SSR marker were placed in the same contig. However, there are cases that need further analysis such as the cases where BAC clones containing SSR markers at distant loci were placed in the same contig. Since the fingerprinting is based only on shared bands of similar size between clones, this could be explained either by duplication events or the presence of repeated sequences such as transposable elements (Klein et al. 2000). BAC end sequencing and reiterative screening of the library could help to eliminate false positive contigs. Alternatively, Klein et al. (2000) used a method based on AFLP technology to build up a whole integrated genetic and physical map of the sorghum genome. This method identified 2,400 BAC clones organized in 700 contigs and 891 unique new markers. To anchor the physical map to the genetic map of maize, three BAC libraries have been constructed and screened with 90 RFLP core markers providing a framework for the physical mapping and sequencing of the maize genome (Yim et al. 2002). In an other work, Yuan et al. (2000) used an in silico strategy to anchor 586 BAC clones to 418 genetic markers of the rice map providing a set of nucleation points throughout the $430 \mathrm{Mb}$ rice genome. However, the sunflower genome is larger $(3,000 \mathrm{Mb}$, Arumuganathan and Earle 1991) and sequencing the ends of all the 150,000 BAC clones should necessitate a huge effort that is beyond the capacity solely of one laboratory. Therefore, the public resource described and the results presented here provide an alternative and simple strategy to provide a framework by detecting seed contigs throughout the sunflower map. The SSR markers described by Tang et al. (2002) are likely to be used in the future in several mapping of agronomic traits in sunflower. Therefore, when an interesting region is identified by SSR mapping, the SSR markers could be used to screen the BAC library and to develop additional markers. The anchoring of the whole set of the 419 sunflower SSR markers is being initiated and the identification of these minimal BAC 'tiling paths' should in turn set the stage for the exploration of targeted genomic regions of interest including the development of markers and positional cloning. 
Acknowledgments This work was supported by a grant from the French consortium Génoplante GOP-HG2. We thank P-J. Ripoll for the helpful discussions and F. Cambon for her technical assistance.

\section{References}

Arumuganathan K, Earle ED (1991) Nuclear DNA content of some important plant species. Plant Mol Biol Rep 9:208-219

Asakawa S, Abe I, Kudoh Y, Kishi N, Wang Y, Kubota R, Kudoh J, Kawasaki K, Minoshima S, Shimizu N (1997) Human BAC library: construction and rapid screening. Gene 191:69-79

Berry ST, Leon AJ, Hanfrey CC, Challis P, Burkholz A, Barnes SJ, Rufener GK, Lee M, Caligari PDS (1995) Molecular-marker analysis of Helianthus annuus L. Construction of an RFLP linkage map for cultivated sunflower. Theor Appl Genet 91:195-199

Bouzidi MF, Badaoui S, Cambon F, Vear F, De Labrouhe DT, Nicolas P, Mouzeyar S (2002) Molecular analysis of a major locus for resistance to downy mildew in sunflower with specific PCRbased markers. Theor Appl Genet 104:592-600

Buckler AJ, Chang DD, Graw SL, Brook JD, Haber DA, Sharp PA, Housman DE (1999) Exon amplification: a strategy to isolate mammalian genes based on RNA splicing. Proc Natl Acad Sci USA 88:4005-4009

Gentzbittel L, Vear F, Zhang Y-X, Berville HA, Nicolas P (1995) Development of a consensus linkage RFLP map of cultivated sunflower (Helianthus annuus L.). Theor Appl Genet 90:10791086

Gentzbittel L, Mestries E, Mouzeyar S, Mazeyrat F, Badaoui S, Vear F, Tourvieille de Labrouhe D, Nicolas P (1999) A composite map of expressed sequences and phenotypic traits of the sunflower (Helianthus annuus L.) genome. Theor Appl Genet 9:218234

Gentzbittel L, Abbott A, Galaud JP, Georgi L, Fabre F, Liboz T, Alibert $\mathrm{G}$ (2002) A bacterial artificial chromosome (BAC) library for sunflower, and identification of clones containing genes for putative transmembrane receptors. Mol Genet Genomics 266:979-987
Klein PE, Klein RR, Cartinhour SW, Ulanch PE, Dong J, Obert JA, Morishige DT, Schlueter SD, Childs KL, Ale M, Mullet JE (2000) A high-throughput AFLP-based method for constructing integrated genetic and physical maps: progress toward a sorghum genome map. Genome Res 10:789-807

Ota T, Amemiya CT (1996) A nonradioactive method for improved restriction analysis and fingerprinting of large P1 artificial chromosome clones. Genet Anal 12:173-178

Ozdemir N, Horn R, Friedt W (2004) Construction and characterization of a BAC library for sunflower (Helianthus annuus L.). Euphyt 138:177-183

Radwan O, Mouzeyar S, Nicolas P, Bouzidi MF (2005) Induction of a sunflower CC-NBS-LRR resistance gene analogue during incompatible interaction with Plasmopara halstedii. J Exp Bot $56: 567-575$

Soderlund C, Longden I, Mott R (1997) FPC: a system for building contigs from restriction fingerprinted clones. CABIOS 13:523535

Soderlund C, Humphray S, Dunham I, French L (2000) Contigs built with fingerprints, markers, and FPC V4.7. Genome Res 11:934-941

Tang S, Yu JK, Slabaugh B, Shintani K, Knapp JS (2002) Simple sequence repeat map of the sunflower genome. Theor Appl Genet 105:1124-1136

Tao Q, Wang A, Zhang HB (2002) One large-insert plant-transformation-competent BIBAC library and three BAC libraries of Japonica rice for genome research in rice and other grasses. Theor Appl Genet 105:1058-1066

Vershinin AV, Druka A, Alkhimova AG, Kleinhofs A, Heslop-Harrison JS (2002) LINEs and gypsy-like retrotransposons in Hordeum species. Plant Mol Biol 49:1-14

Yim YS, Davis GL, Duru NA, Musket TA, Linton EW, Messing JW, McMullen MD, Soderlund CA, Polacco ML, Gardiner JM, Coe EH Jr (2002) Characterization of three maize bacterial artificial chromosome libraries toward anchoring of the physical map to the genetic map using high-density bacterial artificial chromosome filter hybridization. Plant Physiol 130:1686-1696

Yuan Q, Liang F, Hsiao J, Zismann V, Benito MI, Quackenbush J, Wing R, Buell R (2000) Anchoring of rice BAC clones to the rice genetic map in silico.Nucleic Acids Res 28:3636-3641 